\title{
Schätzung von Varianzkomponenten mit Gruppenmittelwerten am Beispiel von Legehennen, gehalten in Gruppenkäfigen
}

\begin{abstract}
Title of the paper: Estimation of variance components from group mean records of laying hens housed in group cages

Two models are presented to estimate variance components if only group mean records are available. The first model accounts for additive genetic relationships and full-sib group effects (SIMIANER and GJERDE, 1991) and the second model contains the additive genetic effects of all animals from one cage by using modified design matrices. Estimates of the genetic parameters were obtained by the MIVQUE-method (RAO, 1971; LAMOTTE, 1973). The variances of the estimated heritabilities were derived from the information matrix. Estimations from individual records and from average records (cage average) were compared in a small application on laying hen data. The analysed trait was single egg weight measured on hens housed in group cages. It could be shown: If cage variance is negligible, than for the estimation of the heritabilities full-sib data can be used successfully. The application of the modified animal model is suggested, because this model can take into account more complex relationships between the animals of one cage.
\end{abstract}

Key Words: MIVQUE, variance components, average performance, group cages, laying hens

\section{Zusammenfassung}

Zur Schätzung von Varianzkomponenten auf der Basis von Gruppenmittelwerten erfasst an Legehennen, gehalten in Gruppenkäfigen, werden 2 Modelle untersucht. Zum einen wird das von SIMIANER und GJERDE (1991) vorgeschlagene Modell mit Vollgeschwistergruppen-Effekten und zum anderen ein Tiermodell mit den additiv genetischen Effekten aller Tiere pro Käfig bei modifizierten Versuchsplanmatrizen genutzt. Die Schätzung der genetischen Parameter erfolgte mit der MIVQUE-Methode (RAO, 1971; LAMOTTE, 1973), wobei die Varianzen der geschätzten Heritabiltäten mit Hilfe der Informationsmatrix abgeleitet wurden. Zum Vergleich der Schätzungen auf der Basis von Einzelleistungen und mittleren Leistungen dienten Datensätze von Legehennen für das Merkmal Eigewicht von in Gruppenkäfigen erfassten Leistungen. Es zeigte sich, dass bei Abwesenheit von Käfigvarianz, die Modelle mit mittleren Leistungen zur Schätzung der Heritabiltät für Einzelleistungen erfasst in Vollgeschwisterkäfigen geeignet sind. Für die Auswertung von Mittelwerten wird das modifizierte Tiermodell, welches auch komplexe verwandtschaftliche Beziehungen innerhalb der Tiergruppen abbilden kann, empfohlen.

Schlüsselwörter: MIVQUE, Varianzkomponenten, mittlere Leistungen, Gruppenkäfig, Legehenne

\section{Einleitung}

In der Leistungsprüfung bei Legehennen ist der Einzelkäfig nach wie vor die bevorzugte Haltungsform um tierindividuelle Daten zu erfassen. Diese Einzeltierinformation ist die Voraussetzung für eine hohe Genauigkeit der Zuchtwertschätzung und Selektion. Allerdings sind bei dieser Form der Leistungsprüfung Genotyp-Umwelt-Interaktionen möglich, weil die praxisübliche Haltung in Gruppenkäfigen stattfindet. In dieser Kleingruppe innerhalb eines Käfigs haben soziale Interaktionen (wie z.B. Konkurrenz 
um Futter und Wasser, Festlegung der Rangordnung usw.) einen Einfluss auf die Leistung der Tiere. Weiterhin können Verhaltensauffälligkeiten wie Federpicken und Kannibalismus auftreten. Um Auswirkungen von möglichen Genotyp-Umwelt-Interaktionen vorzubeugen, werden deshalb Legehennen neben der Einzelkäfigprüfung auch in Gruppenkäfigen einer Leistungsprüfung unterzogen.

Durch die Haltung von mehreren Tieren in einem Käfig sind für viele Merkmale der Eiproduktion nur mittlere Leistungen verfügbar oder es liegen Einzelleistungen vor, die nicht eindeutig einzelnen Tieren zugeordnet werden können. Von SIMIANER und GJERDE (1991) wurden Modelle zur Schätzung der Varianzkomponenten auf der Basis von Mittelwerten, berechnet mit Leistungen von Vollgeschwistergruppen, angegeben. Durch Einführung von genetischen Effekten für jedes Elternpaar und durch Anwendung der MIVQUE-Methode (RAO, 1971; LAMOTTE, 1971) leiteten die Autoren Algorithmen zur Schätzung der additiven und der Dominanzvarianz ab. In vorliegender Arbeit wird die Vorgehensweise von SIMIANER und GJERDE (1991) beibehalten. Die mittleren Leistungen je Käfig werden jedoch mit den genetischen Effekten der Tiere pro Käfig beschrieben. Dies hat den Vorteil, dass bei einer anschließenden Zuchtwertschätzung mit der BLUP-Methode für alle Pedigree-Tiere und nicht nur für die Elternpaareffekte Vorhersagen anfallen und die bekannten Methoden zur Aufstellung und Lösung der Mixed Model Gleichungen (HENDERSON, 1984) anwendbar sind. Für die Untersuchungen standen Einzeleigewichte von Legehennen erfasst in Gruppenkäfigen zur Verfügung. Folglich gestattete das Datenmaterial einen Vergleich der Varianzschätzung auf der Basis von Einzel- und Gruppenleistungen. Ziel der Arbeit ist es zu prüfen, inwieweit Modelle für mittlere Leistungen in der Lage sind, die Heritabilität für in Gruppenkäfige erfasste Einzelleistungen richtig zu schätzen.

2.

Modelle zur Schätzung von Varianzkomponenten mit Leistungen, erbracht in Gruppenkäfigen

Folgende Abkürzungen seien eingeführt:

hre - fixer Haus*Reihen $*$ Etagen-Effekt, c - Käfigeffekt, a - additiv-genetischer Effekt, $\mathrm{d}$ - Dominanzeffekt, $\mathrm{p}$ - Elternpaar-Effekt, e - Resteffekt im Tiermodell, $\varepsilon$ - Resteffekt in den Modellen für Gruppenleistungen, $n$ - Anzahl Tiere pro Käfig

Tabelle 1

Modelle für die Varianzanalysen mit Einzel- und Gruppenleistungen (Models for the analysis of variance with single- and group records)

\begin{tabular}{lllll}
\hline Beob. & Modell & Bez. & Effekte & Varianzen (Interpretation) \\
\hline Einzelne & Tiermodell & T1 & hre, a, d, c, e & $\sigma_{a}^{2}, \sigma_{d}^{2}, \sigma_{c}^{2}, \sigma_{e}^{2}$ \\
& & T2 & hre, a, d, e & $\sigma_{a}^{2}, \sigma_{d}^{2}, \sigma_{e}^{2}$ \\
& & T3 & hre, a, c, e & $\sigma_{a}^{2}, \sigma_{c}^{2}, \sigma_{e}^{2}$ \\
& & T4 & hre, a, e & $\sigma_{a}^{2}, \sigma_{e}^{2}$ \\
\hline $\begin{array}{l}\text { Mittlere } \\
\text { Leistungen }\end{array}$ & Elternpaar- & P1 & hre, p, $\varepsilon$ & $\sigma_{p}^{2}=\left(\frac{1}{2} \cdot \sigma_{a}^{2}\right)$, \\
& modell & & & $\sigma_{\varepsilon}^{2}=\left(\frac{1}{2} \cdot \sigma_{a}^{2}+\sigma_{e}^{2}\right)$ \\
& Tiermodell & T5 & hre, a, $\varepsilon$ & $\sigma_{a}^{2}, \sigma_{\varepsilon}^{2}=\left(\sigma_{e}^{2}\right)$ \\
& & T6 & hre, a, d, $\varepsilon$ & $\sigma_{a}^{2}, \sigma_{d}^{2}, \sigma_{\varepsilon}^{2}=\left(n \sigma_{c}^{2}+\sigma_{e}^{2}\right)$ \\
\hline
\end{tabular}


Da die obigen Tiermodelle für Einzelleistungen aus der einschlägigen Literatur hinreichend bekannt sind, werden lediglich die Modelle basierend auf mittleren Leistungen nachfolgend ausführlicher beschrieben.

\subsection{Das Modell mit Elternpaareffekten für Käfigmittelwerte}

Das von SIMIANER und GJERDE (1991) vorgeschlagene Modell für Mittelwerte von Vollgeschwistergruppen angewendet auf mittlere Leistungen pro Käfig besitzt (ohne Berücksichtigung von Dominanz) in Matrizenschreibweise die Gestalt:

(P1) $\bar{y}=X \beta+Z p+c+\varepsilon$

Hierbei sind:
$\bar{y} \quad-$
Vektor der Käfigmittelwerte
$\beta-\quad$ Vektor der fixen Effekte (im weiteren der kombinierte Effekt aus Haus, Reihe und Etage)
$p \quad-\quad$ Vektor der zufälligen Elternpaareffekte $p_{i}$ (mit $p_{i}$ additiv genetischer Effekt, der Vollgeschwistergruppe $i$ gemeinsam ist)
$c \quad-\quad$ Vektor der zufälligen Käfigeffekte $c_{j}$ (mit $c_{j}$ systematischer Umwelt- effekt, der allen Tieren in einem Käfig gemeinsam ist)
$\varepsilon \quad-\quad$ Vektor der zufälligen Resteffekte für die Käfigmittelwerte
$X, Z \quad-\quad$ Versuchsplanmatrizen der festen und zufälligen Effekte

Für die Varianzkomponenten in Modell (P1) gilt:

$$
\begin{array}{ll}
\operatorname{Var}(p)=A_{p} \cdot \sigma_{p}^{2}=A_{p} \cdot \frac{1}{2} \sigma_{a}^{2} ; & \operatorname{Var}(c)=I_{N} \cdot \sigma_{c}^{2} ; \\
\operatorname{Var}(\varepsilon)=R \cdot \sigma_{\varepsilon}^{2}=R \cdot\left(\frac{1}{2} \sigma_{a}^{2}+\sigma_{e}^{2}\right) ; & \text { mit } \quad R=\operatorname{diag}\left(1 / n_{j}\right) \\
n_{j}-\text { Anzahl Tiere in Käfig } j ; & \mathrm{N}-\text { Anzahl Käfige; } \sigma_{a}^{2} \text { - additiv genetische } \\
\quad \text { Varianz; } & \\
\sigma_{e}^{2}-\text { Restvarianz einer Einzelleistung erfasst im Gruppenkäfig }
\end{array}
$$

Sei der Beobachtungsvektor normalverteilt gemäß $y \approx N(X \beta, V)$, dann besitzt die VarianzKovarianz-Matrix (VC-Matrix) V die Darstellung:

$$
\begin{aligned}
& V=Z A_{p} Z^{\prime} \cdot \sigma_{p}^{2}+I_{N} \cdot \sigma_{c}^{2}+R \cdot \sigma_{\varepsilon}^{2} ; \quad V=V_{1} \cdot \sigma_{p}^{2}+V_{2} \cdot \sigma_{c}^{2}+V_{3} \cdot \sigma_{\varepsilon}^{2} \\
& \text { Sei } \left.\quad S=\left\{s_{i j}\right\}=\left\{\operatorname{tr}\left(V_{i} P V_{j} P\right)\right)\right\} ; \quad r=\left\{r_{i}\right\}=\left\{y^{\prime} P V_{i} P y\right\} ; \\
& \text { mit } P=V^{-1}-V^{-1} X\left(X^{\prime} V^{-1} X\right)^{-} X^{\prime} V^{-1}
\end{aligned}
$$

Dann erhält man MIVQUE-Schätzungen durch iterative Lösung des folgenden Gleichungssystems (SEARLE, 1979):

$$
S(\hat{\theta}) \cdot \hat{\theta}=r(\hat{\theta})
$$$$
\text { mit } \quad \hat{\theta}^{\prime}=\left(\hat{\sigma}_{p}^{2}, \hat{\sigma}_{c}^{2} ; \hat{\sigma}_{\varepsilon}^{2}\right)
$$

Bemerkungen:
a) Sei $n_{j}=n$ für alle $j$, dann folgt $\operatorname{Rg}(S)=\operatorname{Rg}(S, r)$, so dass (1) nicht mehr eindeutig lösbar ist. Folglich müssen in (P1) die Käfigeffekte vernachlässigt werden.
b) Unter Verwendung der Fisherschen Informationsmatrix $I=-E(H)=0.5 \cdot S$ (mit H- Hessianmatrix) ergibt sich für die Schätzfehler:




$$
\operatorname{Var}(\hat{\theta})=[I(\hat{\theta})]^{-1}=2 \cdot S^{-1}(\hat{\theta})
$$

Sei $\hat{\gamma}$ eine erwartungstreue Schätzung für $\gamma(\theta)=\left(\gamma_{1}, \gamma_{2}\right)^{\prime}$ mit $\gamma_{1}=\sigma_{p}^{2}+\sigma_{\varepsilon}^{2}$; und $\gamma_{2}=2 \cdot \sigma_{p}^{2} /\left(\sigma_{p}^{2}+\sigma_{\varepsilon}^{2}\right)$. Setzt man $\Delta_{\theta}=\frac{\partial}{\partial \theta} \gamma(\theta)$, so gilt für die Varianz der transformierten Parameter näherungsweise (vgl. LÄUTER und PINCUS, 1989) :

$$
\operatorname{Var}(\hat{\gamma})=\Delta_{\theta}(\hat{\theta})[I(\hat{\theta})]^{-1} \Delta_{\theta}^{\prime}(\hat{\theta})
$$

c) Die Aufstellung der Matrix $A_{p}$ kann, wie bei SIMIANER und GJERDE (1991) beschrieben, erfolgen. Es sei bemerkt, dass $A_{p}$ im allgemeinen nicht vollen Rang besitzt, also nur positiv semidefinit ist. Eine Rangreduzierung tritt z.B. auf, falls 2 Mütter existieren, die jeweils an 2 Väter angepaart werden. Die zugehörige Matrix der 4 Elternpaareffekte besitzt nachfolgende Gestalt:

$$
A_{p}=\left(\begin{array}{cccc}
1,0 & 0,5 & 0,5 & 0,0 \\
0,5 & 1,0 & 0,0 & 0,5 \\
0,5 & 0,0 & 1,0 & 0,5 \\
0,0 & 0,5 & 0,5 & 1,0
\end{array}\right) ; \quad \text { mit } \quad \operatorname{Rg}\left(A_{p}\right)=3
$$

Tritt in einem Datenmaterial die oben beschriebene nicht hierarchische Zuordnung der Mütter zu den Vätern auf, so kann die übliche Vorgehensweise zur Aufstellung der Mixed Model Gleichungen (MMG) unter Verwendung von $\mathrm{A}_{\mathrm{p}}^{-1}$ nicht verwendet werden. Entsprechende Modifikationen zur Aufstellung der MMG lediglich bei Benutzung der VC-Matrix des Vektors der zufälligen Effekte, ohne deren Inversion, sind z.B. bei SEARLE (1979) zu finden.

\subsection{Tiermodell für Käfigmittelwerte}

Zur besseren Veranschaulichung wird das Tiermodell für mittlere Leistungen in Vektorschreibweise angegeben. Sei $\bar{y}_{i}$ die mittlere Leistung aller Tiere aus Käfig $i$. Dann gilt:

$$
\bar{y}_{i}=x_{i}^{\prime} \beta+z_{i}^{\prime} a+\varepsilon_{i}
$$

Hierbei sind:

$\bar{y}_{i} \quad-\quad$ mittlere Leistung aller Tiere aus Käfig $i$

$\beta \quad-\quad$ Vektor der fixen Effekte

$a \quad-\quad$ Vektor der additiv genetischen Effekte aller Pedigreetiere

$\varepsilon_{i} \quad-\quad$ zufälliger Resteffekt von Käfig $i\left(\right.$ mit $\left.\operatorname{Var}\left(\varepsilon_{i}\right)=\sigma_{c}^{2}+\sigma_{e}^{2} / n_{i}\right)$

$x_{i}\left(z_{i}\right)-\quad$ Versuchsplan-Vektoren der fixen (zufälligen) Effekte $\left(z_{i}^{\prime}=\left(z_{i 1}, \cdots, z_{i p}\right)\right.$ mit $z_{i j}=1 / n_{i}$ falls sich Tier $j$ in Käfig $i$ befindet, Null sonst)

Folglich besitzen die VC-Matrizen der Vektoren $a$ und $\varepsilon$ analog zu Modell (P1) die Darstellung:

$$
\begin{aligned}
& \operatorname{Var}(a)=A \cdot \sigma_{a}^{2} ; \quad \operatorname{Var}(\varepsilon)=I_{N} \cdot \sigma_{c}^{2}+R \cdot \sigma_{e}^{2} \\
& \text { mit } A \text {-numerische } \operatorname{Verwandtschaftsmatrix~und~} R=\operatorname{diag}\left(1 / n_{i}\right)
\end{aligned}
$$

Die Varianzkomponentenschätzung (VKS) mit den Tiermodellen (T1) bis (T6) und für das Elternpaarmodell (P1) erfolgte mit Hilfe der MIVQUE-Methode wie in Abschnitt 
2.1 kurz beschrieben. Die notwendigen Matrizenoperationen zur VKS einschließlich der Berechnung der Schätzfehler wurden unter Verwendung der Matrixprogrammiersprache IML des Statistikpaktes SAS (Ver. 8.2) realisiert.

\section{Beschreibung des Datenmaterials}

Zum Vergleich der Modelle aus Tabelle 1 standen für das Merkmal Eigewicht (EW) Datensätze mit Einzelwägungen für ein Tagesgelege von Hennen, die in Gruppenkäfigen gehalten wurden, zur Verfügung.

Da die erfassten Einzeleigewichte eines Tagesgelege den Hennen eines Käfigs nicht eindeutig zugeordnet werden können, wurden die Daten im ersten Schritt auf Vollgeschwisterkäfige reduziert. Im zweiten Schritt erfolgte die Eliminierung von lediglich 24 Datensätzen mit weniger als drei Beobachtungen (Einzeleigewicht), so dass sich beim Übergang zu Käfigmittelwerten homogene Restvarianzen in den Modellen (P1), (T5) und (T6) ergaben. Die Reduzierung des Datenmaterials auf Vollgeschwisterkäfige und genau 3 Beobachtungen pro Käfig lieferte folgende Datenstruktur:

Tabelle 2

Beschreibende Statistik für das Merkmal Eigewicht (Descriptive statistics of trait egg weight)

\begin{tabular}{lccccccc}
\hline & Anzahl & & & & \multicolumn{3}{c}{ Stat. Maßzahlen } \\
Beobachtungen & Records & Elternpaare & fixe Eff. & Väter & Mütter & $\overline{\boldsymbol{x}}$ & SD \\
\hline Einzelleistg.(EL) & 1488 & 493 & 45 & 81 & 425 & 65.03 & 4.300 \\
Käfigmittel (KM) & 496 & 493 & 45 & 81 & 425 & 65.03 & 3.043 \\
\hline
\end{tabular}

Die 45 fixen Effekte resultieren aus der Aufteilung der Hennen auf 2 Ställe mit unterschiedlicher Reihen- und Etagenanzahl.

\section{Ergebnisse der Varianzkomponentenschätzung (VKS)}

Die Ergebnisse der VKS mit der MIVQUE-Methode sowohl bei Verwendung von Einzel- als auch von Gruppenleistungen zeigt Tabelle 3. Die mit Modell (T1) geschätzten Varianzkomponenten zeigen, dass sowohl der Datenumfang, die Aufteilung der Familien auf die Käfige als auch die Verwandtschaftsstruktur keine simultane Schätzung für die Dominanz- und Käfigvarianz zulassen. Einerseits liegt nur innerhalb der Vollgeschwisterfamilien Dominanzverwandtschaft vor während andererseits die Anzahl der Elternpaare nur sehr geringfügig kleiner ist als die Käfigzahl (s. Tabelle 2). Folglich existieren nur wenige Vollgeschwistergruppen, deren Familienmitglieder auf mehr als einen Käfig aufgeteilt sind. Die VKS mit MIVQUE liefert im Gegensatz zur REML-Methode auch für kleine Stichproben erwartungstreue Schätzungen. Allerdings müssen deshalb auch, wie für Modell (T1) in Tabelle 3 ausgewiesen, negative Schätzungen (hier für die Käfigvarianz) in Kauf genommen werden. In den Modellen (T2) und (T3) werden die Dominanz- und die Käfigvarianz jeweils separat geschätzt. Die zugehörigen Schätzwerte fallen sehr gering aber zumindest positiv aus, wobei die Schätzung für $\sigma_{d}^{2}$ mit 0,279 über dem Schätzwert für die Käfigvarianz liegt. Unter Verwendung von (T1) wurden mit VCE5.0 (KOVAC u.a., 2002) zusätzlich REMLSchätzungen berechnet. Für $\sigma_{c}^{2}(R E M L)$ ergab sich ein Wert von Null während $\sigma_{d}^{2}(R E M L)$ mit 0,2785 geschätzt wurde. Diese Tatsache und die Ergebnisse der Modelle (T1) bis (T3) lassen auf die Abwesenheit von Umwelteffekten, ausschließlich 
verursacht durch die Gruppenkäfighaltung, für das Merkmal Eigewicht schließen. Zur Auswertung des vorliegenden Datenmaterials scheint das rein additive Tiermodell (T4) am besten geeignet. Dieses Modell lieferte Schätzungen für die additiv genetische und die Restvarianz auf annähernd gleichen Niveau wie die erweiterten Modelle (T1) bis (T3) und führte zu dem kleinsten Standardfehler für die Schätzung der Heritabilität unter allen untersuchten Modellen.

Tabelle 3

Varianzkomponentenschätzung mit Tier- und Vollgeschwistermodellen (Estimation of variance components with animal- and full-sib models)

\begin{tabular}{l|l|l|l|l|l|l}
\hline Modell & $\sigma_{a}^{2}( \pm \boldsymbol{s})$ & $\sigma_{f}^{2}( \pm \boldsymbol{s})$ & $\sigma_{\boldsymbol{c}}^{2}( \pm \boldsymbol{s})$ & $\sigma_{\varepsilon}^{2}( \pm \boldsymbol{s})$ & $\sigma_{\boldsymbol{e}}^{2}( \pm \boldsymbol{s})$ & $\boldsymbol{h}^{2}( \pm \boldsymbol{s})$ \\
\hline T1 & $8,003(1,780)$ & $2,507(2,188)$ & $-2,460(2,101)$ & $\times$ & $8,957(1,063)$ & $0,471(0,094)$ \\
T2 & $7,973(1,778)$ & $0,070(0,656)$ & $\times$ & $\times$ & $8,955(1,061)$ & $0,469(0,094)$ \\
T3 & $8,068(1,770)$ & $\times$ & $0,0129(0,649)$ & $\times$ & $8,924(1,059)$ & $0,474(0,093)$ \\
T4 & $8,090(1,316)$ & $\times$ & $\times$ & $\times$ & $8,917(0,974)$ & $0,476(0,065)$ \\
\hline P1 & $8,068(1,770)$ & $\times$ & $\times$ & $12,997(1,858)$ & $8,963(2,558)$ & $0,474(0,121)$ \\
T5 & $8,068(1,770)$ & $\times$ & $\times$ & $8,963(2,558)$ & $8,963(2,558)$ & $0,474(0,121)$ \\
T6 & $8,003(1,780)$ & $2,507(2,188)$ & $\times$ & $1,575(6,337)$ & $8,955=(1,575+3,0 \cdot 2,460)^{*}$ \\
\hline
\end{tabular}

(mit $\sigma_{f}^{2}=0.25 \cdot \sigma_{d}^{2}$ - Varianz der elterlichen Dominanzeffekte, ${ }^{*}$ ) Verwendung von $\sigma_{c}^{2}$ aus (T1)

Mit Hilfe der Modelle (P1), (T5) und (T6) lassen sich Schätzungen für die Varianzkomponenten lediglich unter Verwendung von mittleren Leistungen je Käfig bereitstellen. Das Modell mit Elternpaareffekten und das Tiermodell für mittlere Leistungen liefern nach Durchführung der genetischen Interpretation übereinstimmende Ergebnisse sowohl für die geschätzten Varianzen als auch für deren Standardfehler. Die Schätzungen für die additiv genetische Varianz einschließlich deren Standardfehler und für die Heritabilität in Modell (P1) und (T5) stimmen mit den Ergebnissen von Tiermodell (T3), welches auf Einzelleistungen unter Berücksichtigung von Käfigeffekten basiert, überein. Der höhere Standardfehler für die Restvarianz und die Heritabilität in (P1) und (T5) im Vergleich zu Modell (T3) resultiert aus dem Informationsverlust über die Variabilität innerhalb der Vollgeschwistergruppen. Hinsichtlich der Schätzungen für die dominanzbedingte Familienvarianz und die additive Varianz zeigen die Modelle (T1) und (T6) äquivalente Ergebnisse. Die Interpretation der mit 1,575 geschätzten Restvarianz in Modell (T6) gestaltet sich als schwierig, weil die dazu notwendige Käfigvarianz nicht mit diesem Modell simultan geschätzt werden kann. Setzt man jedoch die negative Schätzung für die Käfigvarianz in die Interpretation der Restvarianz für Käfigmittel gemäß Tabelle 1 ein, so resultiert mit 8,955 ein plausibler Schätzwert.

\section{Diskussion der Modelle für Gruppenmittel}

Das untersuchte Datenmaterial beinhaltet Einzelleistungen erfasst in Gruppenkäfigen. Da die Daten nur aus einer Generation stammen, die Eigewichte für ein Tagesgelege erfasst wurden und nur Gruppenkäfige mit Vollgeschwistern in die Auswertung eingingen, konnte eine zufällige Zuordnung der Einzeleigewichte zu den Hennen eines Käfigs erfolgen. Somit besteht die Möglichkeit zu überprüfen, inwieweit Modelle für mittlere Leistungen in der Lage sind die Heritabilität für das Merkmal Eigewicht bei Erfassung im Gruppenkäfig richtig zu schätzen. Dagegen lässt sich über die Heritabilität für das Merkmal Eigewicht, erfasst in Einzelkäfigen, keine Aussage treffen. Die 
Legehennenhaltung in Gruppenkäfigen kann, z.B. durch die Konkurrenzsituation andere Umwelteinflüsse und damit verbundene Merkmalsausprägungen hervorbringen als die Haltung der Hennen in Einzelkäfigen. Die Abwesenheit von Käfigvarianz liefert keine Aussagen über denkbare, unterschiedliche Umweltvarianzen in beiden Haltungssystemen und über mögliche Genotyp-Umwelt-Interaktionen. Zur Abklärung dieser Fragestellungen sind z.B. Töchter eines Hahnes sowohl in Einzel- als auch in Gruppenkäfigen zu prüfen (vgl. NURGIARTININGSIH u.a., 2002). Aufgrund der Ergebnisse aus Abschnitt 4 liegt jedoch die Schlussfolgerung nahe, dass für die untersuchte Linie die Erfassung des Eigewichtes durch eine gemeinsame Wägung eines Tagesgeleges möglich ist. Geht man davon aus, dass in Einzelkäfighaltung drei Eier pro Henne gewogen werden, so empfiehlt es sich drei Tagesgelege zu wiegen, um vergleichbare $\mathrm{h}^{2}$-Schätzungen zu erhalten. Diese Aussage gilt jedoch nur für den speziellen Fall, das der Gruppeneffekt im Modell vernachlässigt werden kann (vgl. Tab. 3). Die in dieser Arbeit erzielten Ergebnisse für die Gruppenkäfighaltung von Legehennen dürfen nicht verallgemeinert werden. Allerdings sind die in Abschnitt 2 vorgestellten Vorgehensweisen und Modelle auch auf Konstellationen von Gruppenhaltung bei anderen Tierarten anwendbar und bedürfen folglich vor ihrer Anwendung zusätzlicher, separater Untersuchungen.

Hinsichtlich der Modelle, die sich bei der varianzanalytischen Auswertung auf Käfigmittel stützen, lassen sich folgende Schlussfolgerungen ziehen. Aufgrund der Abwesenheit von Käfigeffekten sind die Modelle (P1) und (T5) zur Schätzung der Heritabilität für das Merkmal Eigewicht mit Hilfe von Gruppenleistungen geeignet. Ein geringerer numerischer Aufwand, der bei der Anwendung der MIVQUE-Methode hauptsächlich in der Inversion der Matrix V besteht, muss über einen höheren Standardfehler für die $\mathrm{h}^{2}$-Schätzung erkauft werden. Die Schätzung von Dominanzvarianz mit mittleren Leistungen ist prinzipiell möglich (vgl. SIMIANER und GJERDE, 1991) jedoch für das vorliegende Datenmaterial wie die Ergebnisse mit Modell (T6) zeigen, bedingt durch den geringen Datenumfang, nicht sinnvoll. Die Anwendung des Elternpaarmodelles ist zumindest für nicht hierarchische Zuchtstrukturen eng an die MIVQUE-Methode und an eine mögliche Vorhersage der zufälligen Modelleffekte mit Hilfe von $\mathrm{V}^{-1}$ geknüpft. Der Modellansatz (T5), welcher die genetischen Effekte aller Pedigree-Tiere durch spezielle Wahl der Versuchsplan-Matrix Z in die Berechnungen einbezieht, ist für die Auswertung von mittleren Gruppenleistungen zu empfehlen, weil dieses Modell auch komplexere verwandtschaftlichere Strukturen innerhalb der Gruppen abbilden und die Zuchtwertschätzung durch Aufstellung und Lösung der MMG mit Hilfe der Inversion der numerischen Verwandtschaftsmatrix A erfolgen kann.

\section{Literatur}

HENDERSON, C.R.:

Applications of linear models in animal breeding. University of Guelph (1984)

KOVAC, M.; GROENEVELD, E., GARCIA-CORTES, L.A.:

VCE-5, a package for the estimation of dispersion parameters. Proc. $7^{\text {th }}$ WCGALP (2002) Montpellier

LAMOTTE, L.R.:

Locally best quadratic estimators of variance components. Univ. of Kentucky, Tech. Report 22 (1971) LÄUTER, H.; PINCUS, R.:

Mathematisch-statistische Datenanalyse. Akademie-Verlag Berlin (1989) 
NURGIARTININGSIH, V.; MIELENZ, N.; PREISINGER, R.; SCHMUTZ, M.; SCHÜLER, L.:

Genetic parameters for egg production and egg weight of laying hens housed in single and group cages. RAO, C.R.:

Arch. Tierz., Dummerstorf 45 (2002) 5, 501-508

Minimum variance quadratic unbiased estimation of variance components. J. Mult. Analysis 1 (1971), $445-456$

SEARLE, S.R.:

Notes on variance component estimation: a detailed account of maximum likelihood and kindred methodology. Cornell Univ., Ithaca, New York (1979)

SIMIANER, H.; GJERDE, B.:

Estimating variance components from fullsib group means. J. Anim. Breed. Genet. 108 (1991), 270-279

Eingegangen: 15.05.2003

Akzeptiert: 18.08.2003

Anschriften der Verfasser

Dr. NORBERT MIELENZ, VERONICA NURGIARTININGSIH, MSc.

Univ.-Prof. Dr. habil. LUTZ SCHÜLER

Institut für Tierzucht und Tierhaltung mit Tierklinik der Martin-Luther-Universität

Halle-Wittenberg

Adam-Kuckhoff-Straße 35

D-06108 Halle

E-Mail: mielenz@landw.uni-halle.de

Dr. MATHIAS SCHMUTZ

Lohmann Tierzucht GmbH

Am Seedeich 9-11

D-27450 Cuxhaven 